Portland State University

PDXScholar

2-1-1998

\title{
Specific heat and critical fields of the organic superconductor $\boldsymbol{\beta} "$ "-(BEDT-TTF)2SF5CH2CF2SO3
}

\author{
S. Wanka \\ Universitat Karlsruhe \\ J. Hagel \\ Universitat Karlsruhe \\ D. Beckmanm \\ Universitat Karlsruhe \\ J. Wosnitza \\ Universitat Karlsruhe
}

J. A. Schlueter

Argonne National Laboratory

See next page for additional authors

Follow this and additional works at: https://pdxscholar.library.pdx.edu/chem_fac

Part of the Physics Commons

Let us know how access to this document benefits you.

\section{Citation Details}

Gard, Gary, Wanka, S., Hagel, J., Beckmann, D., Wosnitza, J., Schlueter, J.A., Williams, J.M., Nixon, P.G., and Winter, R.W. (1998) Specific heat and critical fields of the organic superconductor $\beta$ "-(BEDT-

TTF)2SF5CH2CF2SO3, Physical Review B, 57, 3084

This Article is brought to you for free and open access. It has been accepted for inclusion in Chemistry Faculty Publications and Presentations by an authorized administrator of PDXScholar. Please contact us if we can make this document more accessible: pdxscholar@pdx.edu. 


\section{Authors}

S. Wanka, J. Hagel, D. Beckmanm, J. Wosnitza, J. A. Schlueter, Jack M. Williams, P. G. Nixon, Rolf Walter Winter, and Gary L. Gard 


\title{
Specific heat and critical fields of the organic superconductor $\beta^{\prime \prime}$-(BEDT-TTF) ${ }_{2} \mathrm{SF}_{5} \mathrm{CH}_{2} \mathrm{CF}_{2} \mathrm{SO}_{3}$
}

\author{
S. Wanka, J. Hagel, D. Beckmann, and J. Wosnitza \\ Physikalisches Insititut, Universität Karlsruhe, D-76128 Karlsruhe, Germany \\ J. A. Schlueter and J. M. Williams \\ Chemistry and Materials Science Divisions, Argonne National Laboratory, Argonne, Illinois 60439 \\ P. G. Nixon, R. W. Winter, and G. L. Gard \\ Department of Chemistry, Portland State University, Portland, Oregon 97207
}

(Received 16 June 1997)

\begin{abstract}
We report on specific-heat, magnetization, and ac-susceptibility measurements of $\beta^{\prime \prime}$-(BEDT-TTF) ${ }_{2} \mathrm{SF}_{5} \mathrm{CH}_{2} \mathrm{CF}_{2} \mathrm{SO}_{3}$, an organic superconductor with $T_{c}=4.5 \mathrm{~K}$, where BEDT-TTF stands for bis(ethylenedithio)-tetrathiafulvalene. The jump of the specific heat $C$ at $T_{c}$ and the exact form of the specificheat difference between $C$ in the superconducting $(B=0 \mathrm{~T})$ and in the normal $(B=3.5 \mathrm{~T})$ state can be well described by BCS theory with strong coupling. We estimate an electron-phonon coupling parameter $\lambda \approx 1.1$. From measurements of $C$ in magnetic fields we extract the upper critical field $B_{c 2}(T)$. Low-field dcmagnetization measurements were used to determine the temperature dependence of the lower critical field $B_{c 1}$. From $T_{c}$ down to $\sim 3 \mathrm{~K}$ the characteristic suppression of $B_{c 1}^{\perp}(T)$ below the sensitivity limit is observed. [S0163-1829(98)00305-1]
\end{abstract}

\section{INTRODUCTION}

The majority of superconducting organic salts are based on the electron donor molecule bis(ethylenedithio)tetrathiafulvalene (BEDT-TTF) with stochiometry (BEDT-TTF $)_{2} X$, where $X^{-}$is a monovalent chargecompensating anion. Since the discovery of (BEDT-TTF) ${ }_{2} \mathrm{ReO}_{4}$ in 1983 (Ref. 1) the number of organic superconductors in this class has been constantly growing. In these materials, highly conducting BEDT-TTF ${ }^{+0.5}$ cation layers alternate with separating $X^{-}$anion layers. The packing motifs of the BEDT-TTF molecules depend on the anion $X^{-}$and result in different crystallographic phases. ${ }^{2,3}$ Well investigated is the $\kappa$ phase with polymeric anions such as $\mathrm{Cu}(\mathrm{NCS})_{2}^{-}, \mathrm{Cu}\left[\mathrm{N}(\mathrm{CN})_{2}\right] \mathrm{Br}^{-}$, or $\mathrm{Cu}\left[\mathrm{N}(\mathrm{CN})_{2}\right] \mathrm{Cl}^{-} .4$ The last of these has the highest superconducting transition temperature to date, with $T_{c} \approx 13 \mathrm{~K}$ at $\sim 0.3 \mathrm{kbar}$ applied pressure. ${ }^{5}$ Currently, the nature of the superconducting state in these materials is still an open question. The experimental situation is controversial and inconclusive. ${ }^{6,7}$

A promising recent development is the crystallization of BEDT-TTF with large discrete ions such as $M\left(\mathrm{CF}_{3}\right)_{4}^{-}(M$ $=\mathrm{Cu}, \mathrm{Ag}$, or $\mathrm{Au})$ to form superconducting salts, of the general formula $\kappa$-(BEDT-TTF $)_{2} M \mathrm{CF}_{3}(1,1,2$-trihaloethane), which have similar superconducting transition temperatures. ${ }^{8}$ In analogy to these perfluorinated organometallic complex anions another step to create new organic superconductors was the use of large polyfluorinated anions, i.e., $\mathrm{SF}_{5} \mathrm{CH}_{2} \mathrm{CF}_{2} \mathrm{SO}_{3}^{-} \cdot{ }^{9}$ The $\beta^{\prime \prime}$-(BEDT-TTF) ${ }_{2} \mathrm{SF}_{5} \mathrm{CH}_{2} \mathrm{CF}_{2} \mathrm{SO}_{3}$ salt has a superconducting onset temperature of $\sim 5.2 \mathrm{~K}$ according to ac-susceptibility measurements. ${ }^{9}$ To date only limited information on the thermodynamic properties of this first entirely organic superconductor is known.
One important thermodynamic property, which is frequently used to characterize a sample is the specific heat $C$, which yields information on the electronic and the phononic excitation spectra. In addition, the jump height $\Delta C$ at $T_{c}$ and the temperature dependence of $C$ in the superconducting state can be compared to the BCS prediction. We investigated $C$ of a high-quality single crystal of $\beta^{\prime \prime}$-(BEDT-TTF $)_{2} \mathrm{SF}_{5} \mathrm{CH}_{2} \mathrm{CF}_{2} \mathrm{SO}_{3}$ from $\sim 0.7$ to $7 \mathrm{~K}$ in zero and different magnetic fields up to $3.5 \mathrm{~T}$. We determined the Sommerfeld coefficient $\gamma$ of the electrons in the normal state and the temperature dependence of the upper critical field $B_{c 2}$. To further characterize this organic superconductor we measured the low-field magnetization and the ac susceptibility. From these measurements we were able to extract the lower critical field $B_{c 1}$ and the temperature and angular dependence of the irreversibility field, $B_{\text {irr }}$.

\section{EXPERIMENTAL DETAILS}

The investigated platelike single crystal was prepared by an electrochemical process described in detail in Ref. 9. The specific heat was measured with the standard heat-pulse technique in a ${ }^{3} \mathrm{He}$ cryostat that was equipped with a superconducting magnet for fields up to $4 \mathrm{~T}$. The sample consisted of a single crystal with a total mass of $2.4 \mathrm{mg}$ that was broken into four pieces during our investigations. On one piece of the sample we were able to detect Shubnikov-de Haas oscillations, which proves the high quality of the investigated crystal. ${ }^{10}$ The pieces were glued with a small amount of Apiezon $\mathrm{N}$ grease onto a sapphire plate. On the other side of this sample holder an evaporated Au film serves as a sample heater and a calibrated $\mathrm{RuO}_{2}-\mathrm{SMD}$ (surface mounted device) resistor that is glued to the sapphire is used as a thermometer. The temperature was measured with a high-resolution 


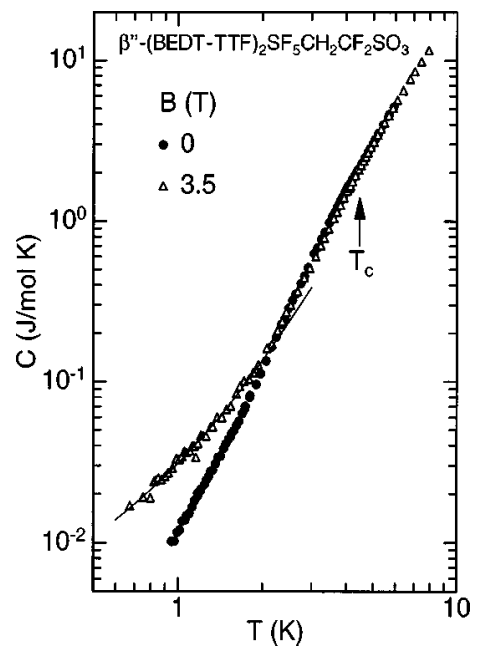

FIG. 1. Specific heat of $\beta^{\prime \prime}$-(BEDT-TTF) ${ }_{2} \mathrm{SF}_{5} \mathrm{CH}_{2} \mathrm{CF}_{2} \mathrm{SO}_{3}$ in zero magnetic field and in $B=3.5 \mathrm{~T}$ applied perpendicular to the highly conducting BEDT-TFF planes. The superconducting transition temperature at $T_{c}$ is marked by an arrow. The solid line is a fit of the form $C=\gamma T+\beta T^{3}$ for the data up to $2 \mathrm{~K}$.

ac resistance bridge that allows a relative temperature resolution of $\delta T / T \approx 10^{-5}$. The heat capacity of the empty sample holder was measured separately in different magnetic fields to obtain a reliable value of the addenda contribution. For $B=0$ the addenda contributed $\sim 80 \%$ at low temperatures $(T \approx 0.9 \mathrm{~K})$, which prohibited a reliable determination of $C$ below this value. At $T_{c}$ the sample contributed approximately $50 \%$ to the total heat capacity, which allowed a determination of $C$ with a relative accuracy $\Delta C / C$ of $\sim 0.5 \%$.

The magnetization $M$ was measured in a ${ }^{4} \mathrm{He}$ croystat down to $2 \mathrm{~K}$ in a low-field superconducting quantum interference device (SQUID) magnetometer. The cryostat is shielded by $\mu$ metal to reduce external fields to below $1 \mu \mathrm{T}$. The ac susceptibility was measured down to $0.45 \mathrm{~K}$ in a ${ }^{3} \mathrm{He}$ cryostat where magnetic fields up to $15 \mathrm{~T}$ can be applied. The ac signal was detected by a compensated pickup coil system with the usual lock-in technique. A modulation-field frequency of $782 \mathrm{~Hz}$ with an amplitude below $0.2 \mathrm{mT}$ was used. For more details see Ref. 11.

\section{RESULTS AND DISCUSSION}

Figure 1 shows the specific heat $C$ of $\beta^{\prime \prime}$-(BEDT-TTF) ${ }_{2} \mathrm{SF}_{5} \mathrm{CH}_{2} \mathrm{CF}_{2} \mathrm{SO}_{3}$ in $B=0$ and $3.5 \mathrm{~T}$ over the whole investigated temperature range in a doublelogarithmic scale. Because of the large phonon background the anomaly in $C$ at $T_{c}$ (marked by an arrow in Fig. 1) is hardly visible. The field of $B=3.5 \mathrm{~T}$ applied perpendicular to the highly conducting BEDT-TTF planes is large enough to suppress the superconducting transition well below the shown temperature range (see below). The low-temperature dependence of the normal-state specific heat up to $2 \mathrm{~K}$ in $B=3.5 \mathrm{~T}$ was approximated by the usual low-temperature fit of the form $C=\gamma T+\beta T^{3}$, where $\gamma$ is the Sommerfeld coefficient of the electronic specific heat and $\beta$ is the coefficient of the low-temperature Debye approximation for the phonon contribution. The coefficients of the fit shown in Fig. 1 are $\gamma=(18.7 \pm 1) \quad \mathrm{mJ} \mathrm{mol}^{-1} \mathrm{~K}^{-2} \quad$ and $\quad \beta=(12.2 \pm 1)$

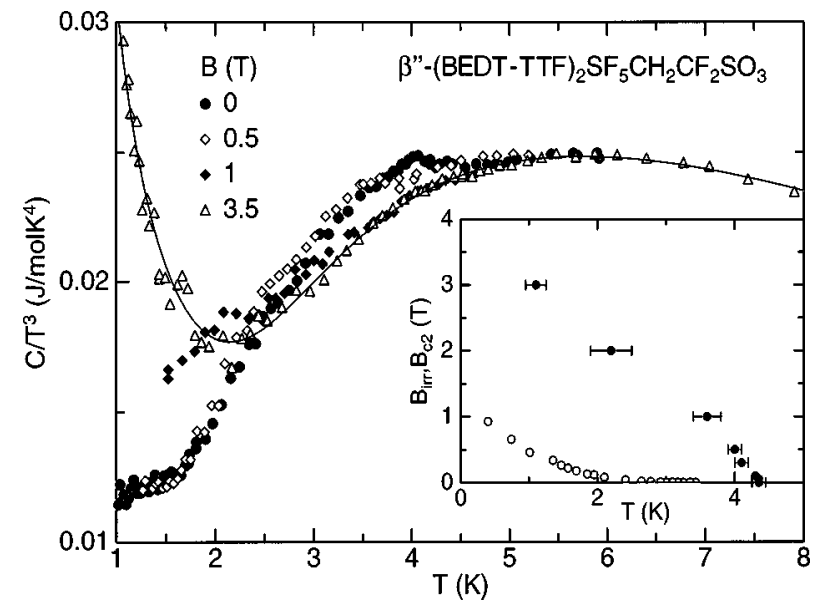

FIG. 2. Plot of the temperature dependence of $C$ divided by $T^{3}$, which allows a better resolution of the specific-heat anomalies and the strong deviation from the simple Debye $T^{3}$ law. The solid line is a fit with the $\gamma$ and $\beta$ as in Fig. 1 plus two Einstein modes (1) as described in the text. The inset shows the temperature dependence of the upper critical field $B_{c 2}$ (solid circles) and the irreversibility field $B_{\text {irr }}$ (open circles) determined by specific-heat and acsusceptibility measurements, respectively.

$\mathrm{mJ} \mathrm{mol}{ }^{-1} \mathrm{~K}^{-4}$. Above $\sim 2 \mathrm{~K}$ a clear upward deviation of the data from the simple Debye $T^{3}$ law is observed that will be discussed later in detail.

The value of the Sommerfeld coefficient $\gamma$ is well within the range reported for other BEDT-TTF superconductors. ${ }^{12-15}$ For a two-dimensional (2D) Fermi liquid the linear coefficient of the specific heat is given by $\gamma^{2 \mathrm{D}}=(\pi / 2)\left(k_{B}^{2} / \hbar^{2}\right) m^{*} V_{M}^{2 \mathrm{D}}$, where $k_{B}$ is the Boltzmann factor, $m^{*}$ is the renormalized effective mass, and $V_{M}^{2 \mathrm{D}}$ is the $2 \mathrm{D}$ molar volume in $\mathrm{m}^{2} / \mathrm{mol}$. With the known room-temperature lattice parameters ${ }^{9}$ and the effective mass from bandstructure calculations $m_{\text {bare }}=1.07 m_{e},{ }^{10} \gamma^{2 \mathrm{D}} \approx 5.4 \mathrm{~mJ} / \mathrm{mol} \mathrm{K}^{2}$ is expected. Comparison with the experimental value hints to an average effective mass enhancement by a factor of $\sim 3.5$. This is somewhat larger than the enhancement found in our $\mathrm{SdH}$ experiment where a cyclotron effective mass of $m_{c}$ $=(1.9 \pm 0.05) m_{e}$ for one small $2 \mathrm{D}$ orbit was found (see also discussion below). ${ }^{10}$ However, besides the closed orbit seen in the $\mathrm{SdH}$ experiment quasi-one-dimensional open bands are predicted that also contribute to the electronic specific heat. Nevertheless, the specific-heat gives additional evidence for a strong mass enhancement due to many-body effects in the title compound.

From the coefficient $\beta$ of the cubic term we obtain a Debye temperature $\theta_{D}=\left(\frac{12}{5} \pi^{4} R n / \beta\right)^{1 / 3}=(221 \pm 7) \quad \mathrm{K}$, where $R$ is the gas constant and $n=68$ is the number of atoms per formula unit. This value is well within the range of $\Theta_{D}$ reported for many other BEDT-TTF compounds. ${ }^{12-15}$ Similar to what was found for $\kappa$-(BEDT-TTF) ${ }_{2} \mathrm{I}_{3}$ (Ref. 12), $\alpha$-(BEDT-TTF) ${ }_{2} M \mathrm{Hg}(\mathrm{SCN})_{4}\left(M=\mathrm{K}, \mathrm{Rb}\right.$, and $\left.\mathrm{NH}_{4}\right)$ (Ref. 14), and other BEDT-TTF salts ${ }^{15}$ an upward deviation from the simple $T^{3}$ law is observed already at low temperatures (see Fig. 1). From Fig. 2 the strong deviation from the Debye law can be seen more clearly (a $T^{3}$ dependence would yield a constant value for $C / T^{3}$ ). The additional phononic contribution with a maximum in $C / T^{3}$ at $\sim 6 \mathrm{~K}$ resembles very 
closely the behavior seen for $\kappa$-(BEDT-TTF $)_{2} \mathrm{I}_{3} .{ }^{12}$ These low-lying phonon excitations are presumably due to librational optical modes which have been observed also by resonant Raman scattering. ${ }^{3,16,17}$ The corresponding excess phonon contribution, $C_{\mathrm{exc}}$, can roughly be described by Einstein-like excitation spectra ${ }^{18}$

$$
C_{\mathrm{exc}}=N_{E} k_{B}\left[\frac{\Theta_{E}}{T}\right]^{2} \frac{\exp \left(\Theta_{E} / T\right)}{\left[\exp \left(\Theta_{E} / T\right)-1\right]^{2}},
$$

where $\Theta_{E}$ is the characteristic Einstein temperature and $N_{E}$ is the number of Einstein modes per mole. With $\gamma$ and $\beta$ as given above and with only one optical mode the best fit to the data gives $\Theta_{E}=28 \mathrm{~K}$ and $N_{E}=1 \times 10^{24} \mathrm{~mol}^{-1}$. However, a much better description is possible by the use of two modes with $\Theta_{E 1}=23 \mathrm{~K}, N_{E 1}=4 \times 10^{23} \mathrm{~mol}^{-1}$ and $\Theta_{E 2}=44 \mathrm{~K}$, $N_{E 2}=1.6 \times 10^{24} \mathrm{~mol}^{-1}$ (solid line in Fig. 2). These Einstein temperatures correspond to mode energies in the range 2 $\mathrm{meV}$ to $3.8 \mathrm{meV}\left(16 \mathrm{~cm}^{-1}\right.$ to $\left.31 \mathrm{~cm}^{-1}\right)$ in rough agreement with the Raman modes found between $20 \mathrm{~cm}^{-1}$ and 50 $\mathrm{cm}^{-13,16,17}$

The specific-heat anomaly of the title compound at $T_{c}$ is rather broad already at $B=0$ (see Fig. 2) ${ }^{19}$ With increasing field the anomaly gets broader and strongly reduced in amplitude. This behavior is known for strongly type-II layered superconductors such as organic metals or cuprates and reflects strong fluctuation effects due to the low dimensionality and the short coherence lengths. From a plot $C / T$ vs $T$ (not shown here) with the usual equal entropy (= area) condition we extract for $B=0, T_{c}=4.4 \mathrm{~K}$ and $\Delta C / \gamma T_{c}=2.1 \pm 0.1$, where $\Delta C$ is the specific-heat jump at $T_{c}$. This latter value is considerably larger than the BCS weak-coupling value of 1.43. We discuss this point later in detail. The specific-heat measurements in different fields allow the extraction of the field dependence of $T_{c}(B)$, i.e., the determination of the upper critical field $B_{c 2}$ for $B$ perpendicular to the BEDT-TTF planes. The result is shown in the inset of Fig. 2. Although some points have rather large error bars due to the weak and broadened $C$ anomalies in these fields we can extrapolate a quite accurate upper critical field at $T=0$ of $B_{c 2}(0)=(3.4$ $\pm 0.4) \mathrm{T}$. This results in an in-plane coherence length of $\xi_{\|}$ $=\sqrt{\Phi_{0} /\left(2 \pi B_{c 2}\right)} \approx 10 \mathrm{~nm}\left(\Phi_{0}=2.07 \cdot 10^{-15} \mathrm{Tm}^{2}\right.$ is the flux quantum), which is well within the range observed for the superconducting BEDT-TTF salts. ${ }^{2,6}$

The inset of Fig. 2 also shows the data for the irreversibility field $B_{\text {irr }}$ determined from field-dependent acsusceptibility measurements. The reversible range between $B_{c 2}$ and $B_{\text {irr }}$ is very large, even for this class of materials. The extrapolated irreversibility field for $T=0$ is of the order $1.5 \mathrm{~T}$. However, neither one of the frequently observed algebraic nor exponential temperature dependences of $B_{\text {irr }}$ can be fitted to the whole temperature range. ${ }^{4,11,20}$ In previous work on other BEDT-TTF materials ${ }^{11,20}$ we found that a rough estimate of the anisotropy can be obtained by the angular dependence of $B_{\text {irr }}$. We, therefore, measured $B_{\text {irr }}$ vs angle at $T=0.4 \mathrm{~K}$ (data not shown). With the use of the Tinkham formula for two-dimensional superconducting thin films ${ }^{21}$ we get a good description of the data with $B_{\text {irr }}^{\perp}=0.93 \mathrm{~T}$ and $B_{\text {irr }}^{\|}=9.5 \mathrm{~T}$ for fields applied perpendicular and parallel to the BEDT-TTF planes, respectively. The resulting anisotropy ra-

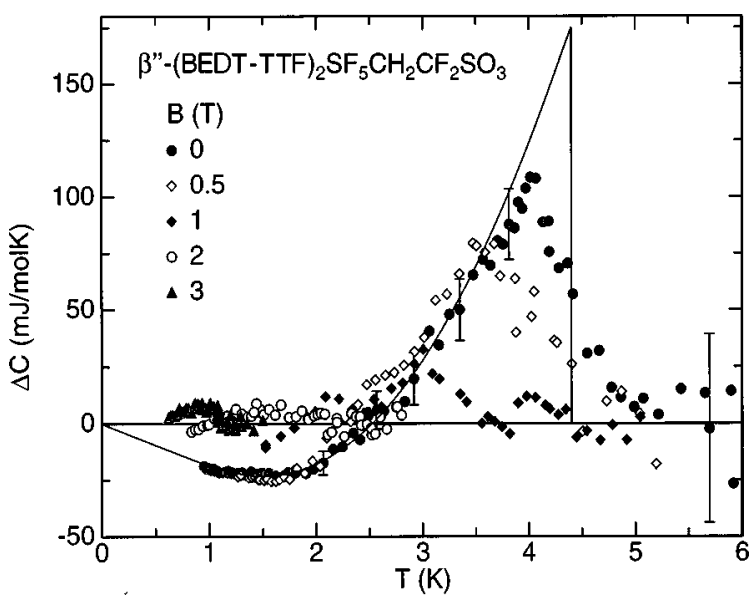

FIG. 3. Specific-heat difference $\Delta C$ between superconducting and normal $C$ for different magnetic fields applied perpendicular to the BEDT-TTF planes. The solid line is a fit of the $B=0$ data according to the BCS theory with strong coupling (Ref. 22).

tio $B_{\mathrm{irr}}^{\|} / B_{\mathrm{irr}}^{\perp} \approx 10$ is rather low. However, additional work is necessary to obtain more information on the superconducting magnetic phase diagram.

In Fig. 3 we have plotted $\Delta C(T)=C_{S}(T)-C_{N}(T)$, the specific-heat difference between $C$ in the superconducting and normal state. In order to get the most reliable estimate for $C_{N}(T)$ the data in $B=3.5 \mathrm{~T}$ were approximated by a polynomial. The field dependences of the $C$ anomalies can be seen now in more detail. As was mentioned, the value of $\Delta C / \gamma T_{c}=2.1 \pm 0.1$ in $B=0$ hints to strong coupling. Another indication for that is the rapidly diminishing electronic contribution to $C$, i.e., the temperature where $\Delta C=0$ is at $\sim 2.5 \mathrm{~K}$ whereas weak-coupling BCS theory predicts a temperature at approximately $T_{c} / 2$. In order to describe $\Delta C(T)$ completely we follow a suggestion of Ref. 22 . The temperature dependence of the energy gap in the superconducting state $\Delta(T)$ is assumed to be BCS like scaled with only one adjustable parameter, the gap ratio $\alpha=\Delta(0) / k_{B} T_{c}$, which is $\alpha_{\mathrm{BCS}}=1.76$ in the weak-coupling case. With $\alpha=2.15$ we get the $\Delta C(T)$ dependence shown by the solid line in Fig. 3, which describes the experimental data perfectly. The deviation of the fit close to $T_{c}$ is because we have not taken into account possible fluctuations. In the weak-coupling case well-known analytic relations between $T_{c}, \Delta(0)$, and $\Delta C / \gamma$ are available. For strong-coupling superconductors these relations are determined by the particular phonon spectrum of the material. The approximative analytic expression $\Delta C / \gamma T_{c}=1.43+f\left(T_{c} / \omega_{\text {ln }}\right)$ has been used to describe this relation. ${ }^{23}$ The function $f$ was determined by a phenomenological fit to exact numerical results from Eliashberg theory where $\omega_{\ln }$ is the average phonon energy. Based on this theory and with the use of the modified McMillan equation ${ }^{24}$

$$
T_{c}=\frac{\omega_{\ln }}{1.2} \exp \left(-\frac{1.04(1+\lambda)}{\lambda-\mu^{*}(1+0.62 \lambda)}\right)
$$

we can estimate the electron-phonon coupling parameter $\lambda$ $=1.1 \pm 0.1$ with the usual assumption of an effective Coulomb repulsion $\mu^{*}=0.1$. This fits nicely with the $\mathrm{SdH}$ results of the title substance ${ }^{10}$ where a cyclotron effective mass of 


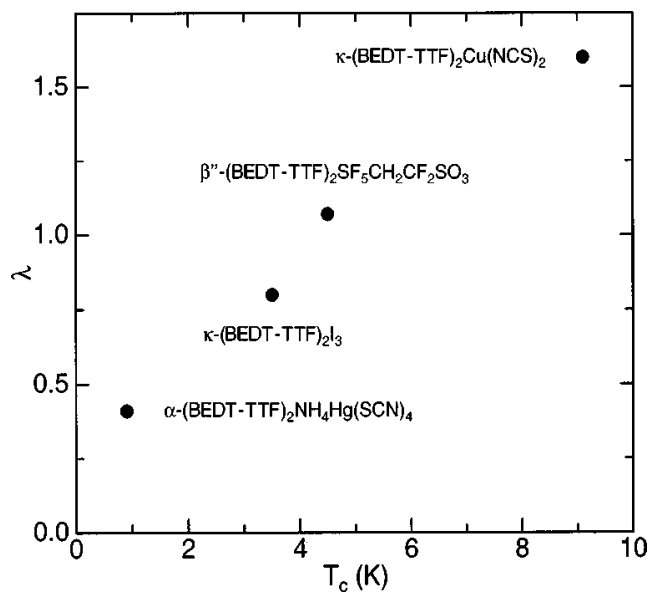

FIG. 4. Electron-phonon coupling constant $\lambda$ for different organic superconductors extracted from specific-heat measurements (Refs. 12-14) as explained in the text.

$m_{c}=(1.9 \pm 0.05) m_{e}$ was found. This value is roughly a factor 1.8 larger than the calculated band-structure cyclotron mass where no many-body effects are incorporated $\left(m_{\text {bare }}\right.$ $\left.=1.07 m_{e}\right) .{ }^{10}$ For the cyclotron mass we cannot disentangle the effect of electron correlations from electron-phonon mass enhancement. From our specific-heat results, however, we may try to estimate the effect of electron-electron interaction $\lambda_{\text {ee }}$. As stated above the Sommerfeld coefficient gives a total effective mass enhancement of 3.5 that may be set equal to $(1+\lambda)\left(1+\lambda_{\mathrm{ee}}\right)$. With $\lambda=1.1$ we estimate an electronelectron interaction $\lambda_{\mathrm{ee}} \approx 0.7$. These results give strong evidence for large many-body effects in $\beta^{\prime \prime}$-(BEDT-TTF) ${ }_{2} \mathrm{SF}_{5} \mathrm{CH}_{2} \mathrm{CF}_{2} \mathrm{SO}_{3}$.

To date only a few high-precision measurements of $C$ of organic superconductors are reported that allow a reliable determination of $\Delta C$ at $T_{c}$. In Fig. 4 the $\lambda$ values for the title substance and three additional superconductors ${ }^{12-14}$ are shown as a function of $T_{c}$. For $\alpha$-(BEDT-TTF $)_{2} \mathrm{NH}_{4} \mathrm{Hg}(\mathrm{SCN})_{4}$ the unmodified McMillan equation $^{25}$ was used, otherwise $\lambda$ was extracted from $\Delta C / \gamma T_{c}$ as described above. For the four different organic superconductors with $T_{c}$ 's between $0.91 \mathrm{~K}$ and $9.1 \mathrm{~K}$ (Refs. 12-14) we find a systematic monotonic increase of $\lambda$ with $T_{c}$. This seems to suggest that the superconductivity in these materials is closely related to the coupling mechanism observed in conventional superconductors. In a recent paper a quasiparticle specific heat quadratic in temperature was reported for the superconducting state of $\kappa$-(BEDT-TTF $)_{2} \mathrm{Cu}\left[\mathrm{N}(\mathrm{CN})_{2}\right] \mathrm{Br}^{26}$ Thereby the authors assumed that the temperature dependence of the phonon specific heat is equal to that of a nonsuperconducting deuterated salt. In contrast, our results for $\beta^{\prime \prime}$-(BEDT-TTF) ${ }_{2} \mathrm{SF}_{5} \mathrm{CH}_{2} \mathrm{CF}_{2} \mathrm{SO}_{3}$ as well as for $\kappa$-(BEDT-TTF) ${ }_{2} \mathrm{I}_{3}$ (Ref. 12) show an exponential temperature dependence of the electronic specific heat, as expected for a BCS superconductor with isotropic gap. ${ }^{27}$ This leaves open the question as to whether the superconducting pairing state might be different within the same class of materials.

As the last point we want to discuss the lower critical field $B_{c 1}$. The usually extremely low $B_{c 1}$ values of organic superconductors require a very sensitive magnetic probe and a good external-field reduction. The inset of Fig. 5 shows the

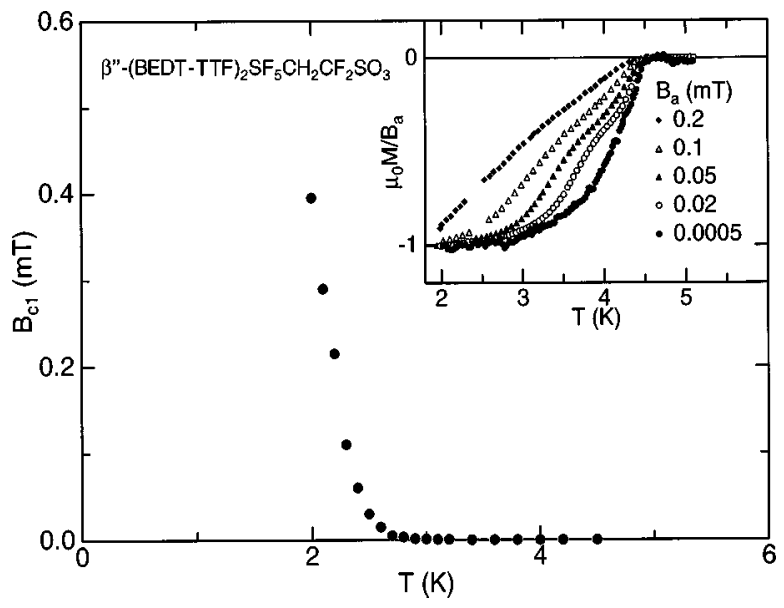

FIG. 5. Temperature dependence of the lower critical field $B_{c 1}$ for $B$ perpendicular to the BEDT-TTF planes. The inset shows the temperature dependence of the magnetization $M$ for different applied fields $B_{a}$.

temperature dependence of the zero-field cooled (= shielding mode) magnetization for the smallest applied field of 0.5 $\mu \mathrm{T}$ and other selected fields (all applied perpendicular to the BEDT-TTF planes). The deviation from ideal diamagnetic behavior, i.e., the first flux penetration into the sample has been used as criterion for the determination of $B_{c 1}$. For details of the procedure see Ref. 11. The main panel of Fig. 5 shows the temperature dependence of $B_{c 1}$ where the demagnetization effect of the platelike sample has been properly taken into account. The most obvious point in this figure is the suppression of $B_{c 1}(T)$ below the detection limit of our apparatus between $T=0.7 T_{c}$ and $T_{c}$. This behavior seems to be a unique feature of layered superconductors. ${ }^{11,20,28} \mathrm{~A}$ theoretical explanation based on fluctuations of individual vortices, ${ }^{29}$ which may explain the suppression of $B_{c 1}$ qualitatively, gives wrong quantitative results. Specifically, the theory predicts a scaling of the suppression range with $T_{c}$ which is not observed for the organic superconductors. ${ }^{11,28}$

By a rough extrapolation of the $B_{c 1}$ data to $T=0$ we obtain $B_{c 1}(0)=(2 \pm 0.5) \mathrm{mT}$. For fields applied parallel to the BEDT-TTF layers the lower critical field was found to be smaller than $6 \mu \mathrm{T}$ down to $2 \mathrm{~K}$. By the use of the relation $B_{c 1}(0) / B_{c 2}(0)=\ln \kappa / 2 \kappa^{2}$ we calculate for $B$ perpendicular to the planes a Ginzburg-Landau parameter $\kappa \approx 59$ and a thermodynamic critical field $B_{\text {cth }}=B_{c 2} /(\kappa \sqrt{2}) \approx 37 \mathrm{mT}$. The large $\kappa$ value is in the upper range reported for organic superconductors. The thermodynamic critical field can be compared with $B_{\text {cth }}$ determined from the specific-heat anomaly. From $\Delta C\left(T_{c}\right)=\left(V_{\mathrm{mol}} T_{c} / \mu_{0}\right)\left(\mathrm{d} B_{\mathrm{cth}} / \mathrm{d} T\right)^{2}$ and by a linear extrapolation of the slope of the thermodynamical critical field $\mathrm{d} B_{\text {cth }} / \mathrm{d} T$ to $T=0$ we find within error bars perfect agreement $\left(B_{\text {cth }} \approx 40 \mathrm{mT}\right)$ with above stated value. $V_{\text {mol }}=5.42$ $\times 10^{-4} \mathrm{~m}^{3}$ is the molar volume and $\mu_{0}$ is the permeability of vacuum.

\section{CONCLUSION}

We presented a study of the thermodynamic properties of the organic superconductor $\beta^{\prime \prime}$-(BEDT-TTF) ${ }_{2} \mathrm{SF}_{5} \mathrm{CH}_{2} \mathrm{CF}_{2} \mathrm{SO}_{3}$. From specific-heat mea- 
surements we found evidence for low-lying optical modes observed frequently in BEDT-TTF-based compounds that are most probably due to optical modes of the BEDT-TTF molecule. The temperature dependence of $\Delta C(T)$ can be very well described by strong-coupling BCS theory. With the modified McMillan formula we estimate a coupling parameter $\lambda \approx 1.1$ that compares well with values obtained for other organic superconductors with different $T_{c}$ 's. We determined the temperature dependence of the upper, lower, and irreversibility field. The title compound is characterized by a very large reversible range in the magnetic phase diagram and by a large Ginzburg-Landau parameter $\kappa$, i.e., a strongly type-II behavior.

\section{ACKNOWLEDGMENTS}

Work at Argonne National Laboratory was supported by the U.S. Department of Energy, Office of Basic Energy Sciences, Divisions of Chemistry and Materials Sciences, under Contract No. W-31-109-ENG-38. Work at Portland State University was supported by NSF (Grant No. Che-9632815) and the Petroleum Research Fund (ACS-PRF Grant No. 31099-AC1).
${ }^{1}$ S. S. P. Parkin, E. M. Engler, R. R. Schumaker, R. Lagier, V. Y. Lee, J. C. Scott, and R. L. Greene, Phys. Rev. Lett. 50, 270 (1983).

${ }^{2}$ T. Ishiguro and K. Yamaji, Organic Superconductors (Springer, Berlin, 1990).

${ }^{3}$ J. M. Williams, J. R. Ferraro, R. J. Thorn, K. D. Carlson, U. Geiser, H.H. Wang, A. M. Kini, and M.-H. Whangbo, Organic Superconductors: Synthesis, Structure, Properties, and Theory (Prentice Hall, Englewood Cliffs, NJ, 1992).

${ }^{4}$ M. Lang, Supercond. Rev. 2, 1 (1996).

${ }^{5}$ J. M. Williams, A. M. Kini, H. H. Wang, K. D. Carlson, U. Geiser, L. K. Montgomery, G. J. Pyrka, D. M. Watkins, J. M. Kommers, S. J. Boryschuk, A. V. Strieby Crouch, W. K. Kwok, J. E. Schriber, D. L. Overmyer, D. Jung, and M.-H. Whangbo, Inorg. Chem. 29, 3272 (1990).

${ }^{6}$ J. Wosnitza, Fermi Surfaces of Low-Dimensional Organic Metals and Superconductors (Springer, Berlin, 1996).

${ }^{7}$ See, e.g., Proceedings of the International Conference of Science and Technology of Synthetic Metals, Snowbird, 1996, edited by Z. V. Vardeny and A. J. Epstein [Synth. Met. 85, 1443-1636 (1997)].

${ }^{8}$ J. A. Schlueter, J. M. Williams, U. Geiser, J. D. Dudek, M. E. Kelly, S. A. Sirchio, K. D. Carlson, D. Naumann, T. Roy, and C. F. Campana, Adv. Mater. 7, 634 (1995).

${ }^{9}$ U. Geiser, J. A. Schlueter, H. H. Wang, A. M. Kini, J. M. Williams, P. P. Sche, H. I. Zakowicz, M. L. VanZile, and J. D. Dudek, J. Am. Chem. Soc. 118, 9996 (1996); J. A. Schlueter, U. Geiser, J. M. Williams, J. D. Dudek, M. E. Kelly, J. P. Flynn, R. R. Wilson, H. I. Zakowicz, P. P. Sche, D. Naumann, T. Roy, P. G. Nixon, R. W. Winter, and G. L. Gard, Synth. Met. 85, 1453 (1997).

${ }^{10}$ D. Beckmann, S. Wanka, J. Wosnitza, J. A. Schlueter, J. M. Williams, P. G. Nixon, R. W. Winter, G. L. Gard, J. Ren, and M.-H. Whangbo, Euro. Phys. J. B (to be published).

${ }^{11}$ S. Wanka, D. Beckmann, J. Wosnitza, E. Balthes, D. Schweitzer, W. Strunz, and H. J. Keller, Phys. Rev. B 53, 9301 (1996).

${ }^{12}$ J. Wosnitza, X. Liu, D. Schweitzer, and H. J. Keller, Phys. Rev. B 50, 12747 (1994).

${ }^{13}$ B. Andraka, J. S. Kim, G. R. Stewart, K. D. Carlson, H. H. Wang, and J. M. Williams, Phys. Rev. B 40, 11345 (1989); J. E. Graebner, R. C. Haddon, S. V. Chichester, and S. H. Glarum, ibid. 41, 4808 (1990).

${ }^{14}$ Y. Nakazawa, A. Kawamoto, and K. Kanoda, Phys. Rev. B 52, 12 890 (1995).

${ }^{15}$ K. Andres, H. Schwenk, and H. Veith, Physica B 143, 334
(1986); G. R. Stewart, J. O'Rourke, G. W. Crabtree, K. D. Carlson, H. H. Wang, J. M. Williams, F. Gross, and K. Andres, Phys. Rev. B 33, 2046 (1986); G. R. Stewart, J. M. Williams, H. H. Wang, L. N. Hall, M. T. Perozzo, and K. D. Carlson, ibid. 34, 6509 (1986); B. Andraka, J. S. Kim, G. R. Stewart, K. D. Carlson, H. H. Wang, and J. M. Williams, ibid. 40, 11345 (1989); B. Andraka, G. R. Stewart, K. D. Carlson, H. H. Wang, M. D. Vashon, and J. M. Williams, ibid. 42, 9963 (1990); B. Andraka, C. S. Jee, J. S. Kim, G. R. Stewart, K. D. Carlson, H. H. Wang, A. V. S. Crouch, A. M. Kini, and J. M. Williams, Solid State Commun. 79, 57 (1991); V. N. Kopylov and A. V. Palnichenko, J. Phys. I 3, 693 (1993).

${ }^{16}$ D. Schweitzer, P. Bele, H. Brunner, E. Gogu, U. Haeberlein, I. Hennig, I. Klutz, R. Swietlik, and H. J. Keller, Z. Phys. B 67, 489 (1987); K. I. Pokhodia, A. Graja, M. Weger, and D. Schweitzer, ibid. 90, 127 (1993).

${ }^{17}$ D. Pedron, G. Visentini, P. Ingenito, R. Bozio, J. M. Williams, and J. A. Schlueter, Synth. Met. 85, 1509 (1997); D. Pedron, G. Visentini, R. Bozio, J. M. Williams, and J. A. Schlueter, Physica C 276, 1 (1997).

${ }^{18}$ See also Ref. 12, where in Eq. (3) the square for $\left(\Theta_{E} / T\right)^{2}$ is missing.

${ }^{19}$ Since the specific-heat cryostat was not shielded against earth's and remnant fields it cannot be excluded that the broadened specific-heat transition at nominal $B=0$ might be due to stray fields (cf. the magnetization curves in the inset of Fig. 5).

${ }^{20}$ J. Hagel, S. Wanka, D. Beckmann, J. Wosnitza, D. Schweitzer, W. Strunz, and M. Thumfart, Physica C (to be published).

${ }^{21}$ M. Tinkham, Phys. Rev. 129, 2913 (1963); M. Tinkham, Introduction to Superconductivity (McGraw-Hill, New York, 1975).

${ }^{22}$ H. Padamsee, J. E. Neighbor, and C. A. Schiffman, J. Low Temp. Phys. 12, 387 (1973).

${ }^{23}$ F. Marsiglio and J. P. Carbotte, Phys. Rev. B 33, 6141 (1986).

${ }^{24}$ P. B. Allen and R. C. Dynes, Phys. Rev. B 12, 905 (1975).

${ }^{25}$ W. L. McMillan, Phys. Rev. 167, 331 (1968).

${ }^{26}$ Y. Nakazawa and K. Kanoda, Phys. Rev. B 55, R8670 (1997).

${ }^{27}$ Note that the strong-coupling fit implies that $C$ is vanishing exponentially towards low temperatures, much faster than any power law or even weak-coupling BCS behavior would do.

${ }^{28}$ J. Wosnitza, S. Wanka, D. Beckmann, J. Hagel, E. Balthes, D. Schweitzer, W. Strunz, and H. J. Keller, Synth. Met. 85, 1557 (1997).

${ }^{29}$ G. Blatter, B. Ivlev, and H. Nordborg, Phys. Rev. B 48, 10448 (1993). 